\title{
Empiric antimicrobial therapy for ventilator-associated pneumonia after brain injury
}

\author{
Antoine Roquilly ${ }^{1}$, Fanny Feuillet ${ }^{2,3}$, Philippe Seguin ${ }^{4}$, Sigismond Lasocki ${ }^{5}$, \\ Raphael Cinotti ${ }^{1}$, Yoann Launey ${ }^{4}$, Lise Thioliere ${ }^{5}$, Ronan Le Floch ${ }^{1}$, \\ Pierre Joachim Mahe ${ }^{1}$, Nicolas Nesseler ${ }^{4}$, Tanguy Cazaubiel ${ }^{1}$, Bertrand Rozec ${ }^{6}$, \\ Didier Lepelletier ${ }^{7}$, Véronique Sebille ${ }^{2,3}$, Yannick Malledant ${ }^{4}$ and \\ Karim Asehnoune for the ATLANREA group ${ }^{1}$
}

\begin{abstract}
Affiliations: 'Intensive Care Unit, Anaesthesia and Critical Care Dept, Hôtel Dieu - HME, University Hospital of Nantes, Nantes, France. ${ }^{2}$ Plateforme de Biométrie, Cellule de promotion de la recherche clinique, University Hospital of Nantes, Nantes, France. ${ }^{3}$ EA 4275 SPHERE "Biostatistics, Pharmacoepidemiology \& Human Science Research", UFR des Sciences Pharmaceutiques, Nantes University, Nantes, France. "Intensive Care Unit, Anaesthesia and Critical Care Dept, University Hospital of Rennes, Rennes, France. ${ }^{5}$ Intensive Care Unit, Anaesthesia and Critical Care Dept, University Hospital of Angers, Nantes, France. 'Intensive Care Unit, Anaesthesia and Critical Care Dept, Laennec, University Hospital of Nantes, Nantes, France. ${ }^{7}$ Infection Control Unit, Hôtel Dieu - HME, University Hospital of Nantes, Nantes, France.
\end{abstract}

Correspondence: Karim Asehnoune, CHU de Nantes, Service d'Anesthésie Réanimation, 1 place Alexis Ricordeau, 44093 Nantes Cedex 1, France. E-mail: karim.asehnounedchu-nantes.fr

ABSTRACT Issues regarding recommendations on empiric antimicrobial therapy for ventilatorassociated pneumonia (VAP) have emerged in specific populations.

To develop and validate a score to guide empiric therapy in brain-injured patients with VAP, we prospectively followed a cohort of 379 brain-injured patients in five intensive care units. The score was externally validated in an independent cohort of 252 brain-injured patients and its extrapolation was tested in 221 burn patients.

The multivariate analysis for predicting resistance (incidence 16.4\%) showed two independent factors: preceding antimicrobial therapy $\geqslant 48 \mathrm{~h}(\mathrm{p}<0.001)$ and VAP onset $\geqslant 10$ days $(\mathrm{p}<0.001)$; the area under the receiver operating characteristic curve (AUC) was 0.822 (95\% CI 0.770-0.883) in the learning cohort and 0.805 (95\% CI $0.732-0.877)$ in the validation cohort. The score built from the factors selected in multivariate analysis predicted resistance with a sensitivity of $83 \%$, a specificity of $71 \%$, a positive predictive value of $37 \%$ and a negative predictive value of $96 \%$ in the validation cohort. The AUC of the multivariate analysis was poor in burn patients (0.671, 95\% CI 0.596-0.751).

Limited-spectrum empirical antimicrobial therapy has low risk of failure in brain-injured patients presenting with VAP before day 10 and when prior antimicrobial therapy lasts $<48 \mathrm{~h}$.

@ERSpublications

To stop spread of resistant bacteria, we should limit broad antimicrobial therapy in brain-injured pneumonia patients http://ow.ly/UL3pS

For editorial comment see Eur Respir J 2016; 47: 1060-1061 [DOI: 10.1183/13993003.02190-2015].

This article has supplementary material available from erj.ersjournals.com

Received: Aug 082015 | Accepted after revision: Nov 10 2015 | First published online: Jan 072016

Support statement: This work was supported by institutional funds.

Conflict of interest: Disclosures can be found alongside the online version of this article at erj.ersjournals.com

Copyright OERS 2016 


\section{Introduction}

Ventilator-associated pneumonia (VAP) is one of the most frequent complications and the main cause of antibiotics use in intensive care units (ICUs) [1]. Early administration of empiric antibiotic therapy is recommended because morbidity, mortality and medical costs increase when the treatment is delayed [2]. Given the risk of widespread bacterial resistance associated with unnecessary use of broad-spectrum antibiotic therapy [3], it is important to balance the individual risk of treatment failure and the community risk of unnecessary broad-spectrum treatment [4].

In 2005, the American Thoracic Society (ATS) and the Infectious Diseases Society of America (IDSA) recommended restricting the use of broad-spectrum antibiotics to patients with a high risk of drug-resistant pathogens, i.e. with one or more risk factors: immune suppression, healthcare-associated pneumonia, antimicrobial therapy in the preceding 3 months and current hospitalisation of $\geqslant 5$ days [5]. However, unjustified broad-spectrum empirical therapy is still common in Europe [6] and issues regarding the validity of these recommendations have emerged. Notably, the strict application of the ATS/IDSA criteria to unselected ICU populations considered at risk of resistance has been associated with an increased rate of death [7].

We and others have previously proposed that some specific conditions, such as trauma, are associated with a low risk of drug-resistant bacteria [8,9]. The rate of VAP reaches up to $40 \%$ for brain-injured patients hospitalised in ICUs [10-12]. An optimisation of the current practice for antimicrobial therapy in this population could therefore have a significant impact. The main objective of this study was to develop and validate a bedside scoring system to predict resistance to antimicrobial therapy in brain-injured patients presenting with VAP. The second objective was to confirm that the initial condition on ICU admission significantly alters the risk of resistance of bacteria involved in VAP and we thus evaluated this using the simplified bedside scoring system in an extrapolation cohort of severe burn patients.

\section{Methods}

\section{Study design and setting}

From January 2007 to March 2013, a prospective, observational, multicenter study was conducted in five ICUs of three university hospitals from the ATLANREA study group, in France. This report follows the STROBE statement for observational studies [13].

\section{Ethics and consent}

The local ethics committee of the University Hospital of Nantes approved the study protocol (Groupe Nantais d'Ethique dans le Domaine de la Santé, Nantes, France). The need for written consent was waived in this epidemiological study because of its observational design. The study was performed in accordance with the ethical standards laid down in the 1964 Declaration of Helsinki and its later amendments.

\section{Learning and validation cohorts: brain-injured patients}

Brain-injured patients developing an episode of VAP were included in the participating ICUs. Brain injury was considered as a Glasgow Coma Scale score $\leqslant 12$ associated with an anomaly related to an acute process on a brain computed tomography (CT) scan [11]. Comatose patients with a normal brain CT scan were not included.

\section{Extrapolation cohort: severe burn patients}

Patients with a burn size $\geqslant 10 \%$ of the total cutaneous surface developing an episode of VAP were recruited in a specialised burn ICU admitting all severe burn patients in the geographic area of the five participating ICUs [14].

\section{Exclusion criteria}

Exclusion criteria were age younger than 18 years or refusal to participate after information was provided.

\section{Definition of ventilator-associated pneumonia}

The definition of VAP followed the international guidelines $[5,15]$. Pneumonia was considered when at least two signs (body temperature $>38^{\circ} \mathrm{C}$; leukocytosis $>12000 \mathrm{~mL}^{-1}$ or leukopenia $<4000 \mathrm{~mL}^{-1}$; purulent pulmonary secretions, increase in daily minimum inspiratory oxygen fraction of $\geqslant 10 \%$ ) were associated with the appearance of a new infiltrate or changes in an existing infiltrate on chest radiograph. Respiratory tract samples using a quantitative culture (threshold of $10^{4} \mathrm{CFU} \cdot \mathrm{mL}^{-1}$ ) for a bronchoalveolar lavage, $10^{3} \mathrm{CFU} \cdot \mathrm{mL}^{-1}$ for a nonbronchoscopic sample or protected specimen brush and $10^{6} \mathrm{CFU} \cdot \mathrm{mL}^{-1}$ for a tracheal sample) were harvested before modification or introduction of any new antimicrobial therapy. Only episodes with positive cultures were counted as VAP. VAP was defined as pneumonia developing after $48 \mathrm{~h}$ of mechanical ventilation and no later than $48 \mathrm{~h}$ following extubation. 
Significant healthcare in the previous 90 days was considered in the case of hospitalisation for $\geqslant 2$ days in the preceding 90 days, or residence in a nursing home or extended care facility, home infusion therapy including antibiotics, home wound care or chronic dialysis within 30 days [16, 17].

\section{Definition of resistance to limited-spectrum antimicrobial therapy}

Limited-spectrum antimicrobial therapy was defined by the ATS/IDSA as the use of ampicillin/sulbactam, ceftriaxone or levofloxacin [5]. Resistance to limited-spectrum antimicrobial therapy was considered when a bacterial strain was resistant to one or more of these antibiotics. In VAP involving at least two strains, resistance was considered as soon as one bacteria was not susceptible to limited-spectrum antimicrobial therapy. Antimicrobial resistance was defined according to the European Committee on Antimicrobial Susceptibility Testing for all the bacteria with a load higher than a pre-specified threshold. Following ATS/ IDSA guidelines [5], Serratia marcescens and Enterobacter species with low minimal inhibitory concentrations for ampicillin/sulbactam, ceftriaxone and levofloxacin $\left(\leqslant 4, \leqslant 1\right.$ and $\leqslant 2 \mathrm{mg} \cdot \mathrm{mL}^{-1}$, respectively) were considered susceptible to treatment with narrow-spectrum antimicrobial therapy.

Inappropriate empirical therapy was considered when at least one bacteria was resistant to the empirical treatment.

\section{Preventive strategies for VAP}

All the participating ICUs routinely applied the following care to prevent VAP: selective oropharyngeal antiseptic decontamination, monitoring of the tracheal cuff pressure, semirecumbent positioning and early enteral feeding. Early tracheotomy, subglottic secretion drainage and selective digestive decontamination were not used. Following French recommendations, antibiotic prophylaxis ( $\leqslant 2$ days) was used in the case of surgery and/or contaminated wounds.

\section{Data collection}

Demographic data, clinical parameters at VAP onset and outcomes were prospectively collected during ICU hospitalisation. The first episode of VAP was the only one considered in patients presenting with multiple infections during ICU hospitalisation.

\section{Statistical analysis}

$60 \%$ of brain-injured patients were randomly assigned to a learning cohort and $40 \%$ were assigned to a validation cohort. This random assignment allowed us to assess the stability and the robustness of results in the validation cohort without any a priori knowledge of the allocation of patients to one cohort or another [18].

First, we identified potential risks factors in the learning cohort by univariate analysis with a cut-off point at 0.20 for the p-value. Criteria collected after VAP diagnosis (e.g. total duration of mechanical ventilation or of hospitalisation, death) were not considered relevant to help clinicians to select a priori an optimal antimicrobial therapy and thus were not included in the multivariate analysis. Second, receiver operating characteristic (ROC) curves were constructed to determine the best cut-offs for quantitative factors selected by the univariate analysis (duration of antimicrobial therapy prior to VAP onset, time between hospitalisation and VAP onset). Statistical indices corresponding to each possible value and clinical considerations (use of discontinuous rather than continuous variables) were taken into account for simplified clinical application and efficiency. Third, variables identified as potential risk factors in univariate analyses were included in a multivariate logistic model in which a backward selection was subsequently applied. Fourth, to validate this predictive model: 1) internal validity was estimated in the learning cohort, 2) reproducibility was estimated in the validation cohort (external validity) and 3) transportability was estimated in the extrapolation cohort of burn patients (external validity). Discrimination was evaluated using the area under the ROC curve (AUC) and its 95\% confidence interval, and calibration was assessed using the Hosmer-Lemeshow test. Finally, an easy-to-use score was constructed with the variables identified in the predictive model (which was similar for the learning and validation cohorts). We considered that patients with no risk factor as at low risk of drug-resistant bacteria and other patients at high risk. The discriminative ability of the simplified score (sensitivity, specificity, positive predictive value and negative predictive value) was estimated in the three cohorts to externally validate the simplified score.

Continuous variables were expressed as median (interquartile range (IQR)) for nonparametric data or mean \pm SD for parametric data. Qualitative variables were expressed as n (\%). The significance level was set at $\mathrm{p} \leqslant 0.05$. Analyses were performed with SAS statistical software version 9.3 (SAS Institute, Cary, NC, USA). 


\section{Results}

Brain-injured populations

During the study period, 631 brain-injured patients were included (379 in the learning cohort, 252 in the validation cohort; figure 1). The distribution of the bacteria responsible for VAP is described in the table 1 . In the learning and validation cohorts, $62(16.4 \%)$ and $42(16.7 \%)$ patients, respectively, presented with resistant bacteria to limited-spectrum antimicrobial therapy. Out of the 631 brain-injured patients, 34 (5.4\%) received inappropriate empirical therapy. Inappropriate empirical therapy was not associated with an increased duration of mechanical ventilation $(\mathrm{p}=0.46)$ and had no significant effect on the rate of mortality $(\mathrm{p}=0.81)$ (table 2$)$.

\section{Univariate analysis: selection of the potential risk factors associated with resistance}

The demographic characteristics and the clinical findings at the time of VAP diagnosis are described in tables 3 and 4, respectively. The rate of significant healthcare in the previous 90 days and the criteria used for the diagnosis of VAP (white blood cell number, arterial oxygen tension/inspiratory oxygen fraction ratio and chest radiograph) did not differ between susceptible and resistant bacteria, and were thus not included in the multivariate analysis. In the univariate analysis, the factors potentially associated with drug resistance were: neurosurgery prior to ICU admission $(\mathrm{p}=0.02)$, mechanical ventilation on admission $(\mathrm{p}=0.11)$, body temperature $(\mathrm{p}=0.08)$, duration of antimicrobial administration prior to VAP diagnosis (including prophylaxis and/or curative treatment, $\mathrm{p}<0.001$ ), and median time between hospitalisation and VAP onset $(\mathrm{p}<0.001)$. These criteria were thus included in the multivariate analysis, which served to build an easy-to-use score.

\section{Multivariate analysis: independent risk factors for bacteria resistant to limited-spectrum} antimicrobial therapy

In an attempt to simplify the use of the results in clinical practice, the duration of prior antimicrobial administration and the time between hospitalisation and VAP onset were dichotomised in two categories (above or below a threshold). The cut-offs of antimicrobial duration during current hospitalisation and of hospitalisation ( $48 \mathrm{~h}$ and 10 days, respectively) were selected because they provide the best sensitivity/ specificity balance (ROC curves; online supplementary figure S1) and the best calibration of the multivariate analysis (Hosmer-Lemeshow test $\mathrm{p}$-value $>0.05$, online supplementary table $\mathrm{S} 1$ ).

The final multivariate analysis selecting the factors independently associated with resistance to limited-spectrum antimicrobial therapy retained a duration of antimicrobial administration prior to VAP diagnosis $\geqslant 48 \mathrm{~h}$ during current hospitalisation (OR 12.63, 95\% CI 6.64-24.03) and a VAP onset $\geqslant 10$ days (OR 2.45, 95\% CI 1.23-4.89). The AUC for the prediction of resistance to limited-spectrum antimicrobial therapy was 0.822 (95\% CI 0.770-0.883; Hosmer-Lemeshow test p-value 0.26) versus 0.735 (95\% CI 0.697-0.786; Hosmer-Lemeshow test p-value 0.61) when the ATS/IDSA criteria were used for predicting resistance (online supplementary figure S2).

\section{External validation in brain-injured patients}

The multivariate analysis presented good characteristics with high AUC and we thus aimed to test its robustness in an independent cohort of brain-injured patients. There were no differences in the characteristics on ICU admission between the learning and the validation cohorts (tables 3 and 4). In the validation cohort, the AUC for the prediction of resistance was 0.805 (95\% CI 0.732-0.877;
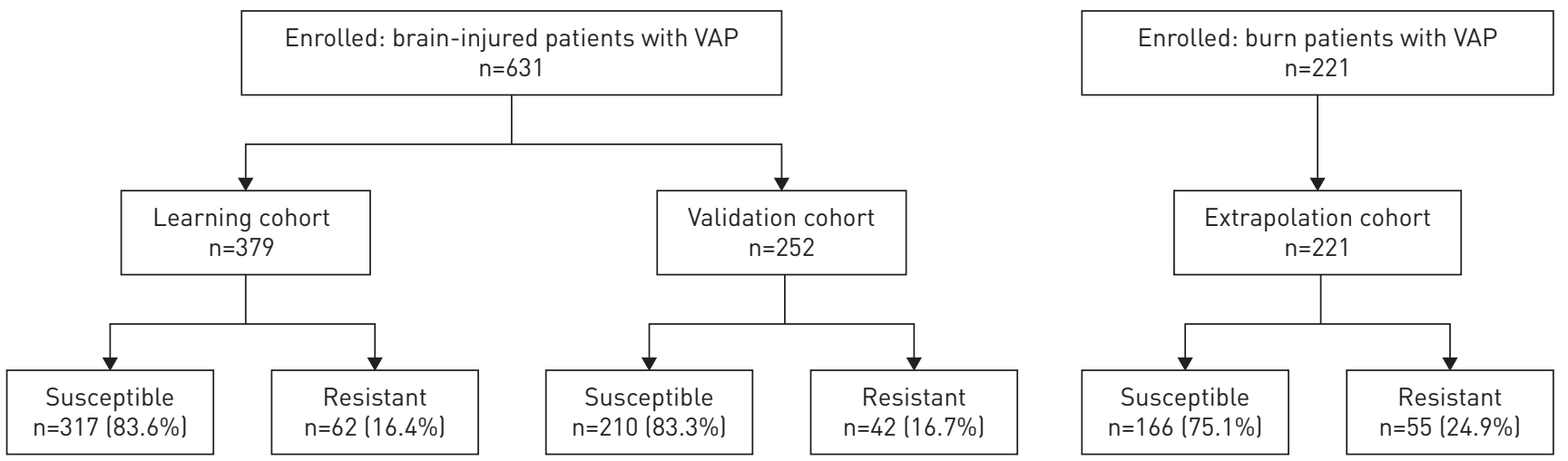

FIGURE 1 Patient flow diagram. VAP: ventilator-associated pneumonia. Bacteria were defined as resistant or susceptible to limited-spectrum antimicrobial therapy (ampicillin/sulbactam, ceftriaxone or levofloxacin). 
TABLE 1 Pathogens involved in ventilator-associated pneumonia (VAP) in the brain-injured population

Learning cohort

Validation cohort

Bacteria susceptible to limited-spectrum antimicrobial therapy
Meticillin-sensitive Staphylococcus aureus
Streptococcus pneumoniae
Haemophilus influenzae
Escherichia coli
Enterobacter species"
Klebsiella pneumonia
Proteus species
Serratia marcescens
Others
Bacteria resistant to limited-spectrum antimicrobial therapy ${ }^{\#}$
Meticillin-resistant Staphylococcus aureus
Streptococcus pneumoniae with decreased susceptibility
to penicillin
Pseudomonas aeruginosa
Acinetobacter species
Enteric Gram-negative bacilli with acquired penicillinase ${ }^{+}$
Enteric Gram-negative bacilli with inducible cefalosporinase ${ }^{\S}$
Others

Data are presented as $n .{ }^{*}$ : ampicillin/sulbactam, ceftriaxone or levofloxacin; ${ }^{\text {9: }}$ according to the American Thoracic Society/Infectious Diseases Society of America guidelines for VAP [5], Serratia marcescens and Enterobacter species were considered susceptible to treatment with narrow-spectrum antimicrobial therapy, except if resistance was found according to European Committee on Antimicrobial Susceptibility Testing guidelines; ${ }^{+}$: resulting in resistance to ampicillin/sulbactam; ${ }^{\S}$ : Enterobacter cloacae, Citrobacter freundii, Hafnia alveii and Providencia species.

TABLE 2 Demographical characteristics and outcomes of brain-injured patients with appropriate or inappropriate empirical antimicrobial therapy for ventilator-associated pneumonia (VAP)

\begin{tabular}{lccc} 
& Appropriate & Inappropriate & p-value \\
\hline Subjects & 597 & 34 & \\
Age years & $48(31-61)$ & $46.5(35-62)$ & 0.90 \\
Male & $434(72.7)$ & $23(67.7)$ & 0.52 \\
Previous hospitalisation in the last 3 months & $113(19.1)$ & $3(8.8)$ & 0.13 \\
Initial diagnosis & & & 0.36 \\
$\quad$ Traumatic brain injury & $329(55.1)$ & $24(70.6)$ & \\
$\quad$ Subarachnoid haemorrhage & $143(24.0)$ & $7(20.6)$ & $2(5.9)$ \\
$\quad$ Stroke & $73(12.2)$ & $1(2.9)$ & \\
$\quad$ Other & $52(8.7)$ & $40(25-49)$ & 0.48 \\
SAPS-II & $40.5(32-49)$ & $8.5(5.5-10)$ & 0.96 \\
SOFA & $8(6-10)$ & $8(4-12)$ & 0.19 \\
Glasgow Coma Scale score on admission & $7(4-9)$ & $12(35.3)$ & 0.98 \\
Significant healthcare in last 90 days & $212(35.5)$ & $5.9 \pm 3.3$ & 0.08 \\
Time between hospitalisation and VAP onset days & $7.0 \pm 4.8$ & $2(5.9)$ & 0.03 \\
Time between hospitalisation and VAP onset $\geqslant \mathbf{1 0}$ days yes & $127(21.3)$ & & \\
Antimicrobial administration prior to VAP onset during & & & \\
$\quad$ current hospitalisation & & $29(85.3)$ & $<0.01$ \\
Yes & $367(61.6)$ & $48(24-72)$ & 0.81 \\
$\quad$ Duration h & $12(12-72)$ & $18(52.9)$ & $<0.001$ \\
Antimicrobial administration prior to VAP onset $\geqslant 48$ h yes & $143(24.0)$ & $20.0 \pm 14.4$ & 0.46 \\
Duration of mechanical ventilation days & $18.3 \pm 12.8$ & $24.9 \pm 15.2$ & 0.86 \\
Duration of ICU hospitalisation days & $25.5 \pm 17.5$ & $6(17.7)$ & 0.81 \\
In ICU death & $96(16.1)$ & & \\
\hline
\end{tabular}

Data are presented as $n$, median (interquartile range), $n(\%)$ or mean \pm SD, unless otherwise stated. SAPS-II: new simplified acute physiology score; SOFA: sequential organ failure assessment; ICU: intensive care unit. 
TABLE 3 Patient characteristics in the learning and validation cohorts according to the presence of bacteria resistant to limited-spectrum antimicrobial chemotherapy

\begin{tabular}{|c|c|c|c|c|c|c|}
\hline & \multicolumn{3}{|c|}{ Learning cohort } & \multicolumn{3}{|c|}{ Validation cohort } \\
\hline & Susceptible & Resistant & p-value & Susceptible & Resistant & p-value ? \\
\hline Subjects & 317 & 62 & & 210 & 42 & \\
\hline Male & $225(71.0)$ & $43(69.4)$ & 0.80 & $158(75.2)$ & $31(73.8)$ & 0.84 \\
\hline Medical history of corticotherapy & $18(5.7)$ & 2 (3.2) & 0.55 & $9(4.3)$ & $2(4.8)$ & 1.00 \\
\hline Immune suppression (active cancer, HIV) & $7(2.2)$ & $1(1.6)$ & 1.00 & $4(1.9)$ & $1(2.4)$ & 1.00 \\
\hline Traumatic brain injury & $170(53.6)$ & 39 (62.3) & & $118(56.2)$ & $26(61.9)$ & \\
\hline Subarachnoid haemorrhage & 80 (25.2) & $14(22.6)$ & & $50(23.8)$ & $6(14.3)$ & \\
\hline Stroke & $40(12.6)$ & $4(6.5)$ & & 27 (12.9) & $4(9.5)$ & \\
\hline Other & $27(8.5)$ & $5(8.1)$ & & $15(7.1)$ & $6(14.3)$ & \\
\hline SAPS-II & $41(31-50)$ & $43.5(28-49)$ & 0.86 & $40(33-48)$ & $40(29-46)$ & 0.82 \\
\hline SOFA & $8(6-10)$ & $8(6-10)$ & 0.63 & $8(6-10)$ & $8(4.5-10)$ & 0.91 \\
\hline Duration of mechanical ventilation days & $17.4 \pm 11.8$ & $22.1 \pm 15.7$ & 0.03 & $17.8 \pm 13.1$ & $23.4 \pm 17.8$ & 0.01 \\
\hline Duration of ICU hospitalisation days & $23.8 \pm 16.1$ & $29.0 \pm 17.7$ & 0.02 & $25.6 \pm 18.7$ & $31.3 \pm 16.7$ & 0.07 \\
\hline In ICU death & $53(16.7)$ & $8(12.9)$ & 0.45 & $37(17.6)$ & $4(9.5)$ & 0.19 \\
\hline \multicolumn{7}{|c|}{$\begin{array}{l}\text { Data are presented as } \mathrm{n} \text {, median (interquartile range), } \mathrm{n}(\%) \text { or mean } \pm \mathrm{SD} \text {, unless otherwise stated. SAPS-II: new simplified acute physiology score; } \\
\text { SOFA: sequential organ failure assessment; ICU: intensive care unit. }{ }^{*}: \text { ampicillin/sulbactam, ceftriaxone or levofloxacin; }{ }^{\uparrow}: \mathrm{p} \text {-values for comparison } \\
\text { between susceptible and resistant to limited-spectrum antimicrobial therapy; }{ }^{+}: \text {significant healthcare (hospitalisation for } \geqslant 2 \text { days in the preceding } \\
90 \text { days, home wound care or residence in an extended care facility, home infusion therapy including antibiotics, or chronic dialysis within } 30 \text { days). }\end{array}$} \\
\hline
\end{tabular}

Hosmer-Lemeshow test p-value 0.49) versus 0.762 (95\% CI 0.713-0.825; Hosmer-Lemeshow test p-value 0.93 ) with the ATS/IDSA guidelines (online supplementary figure S2). The results of the multivariate and the clinical values of the score in the whole population of 631 brain-injured patients are provided in online supplementary table S2.

Definition of an easy-to-use score

The robustness of the multivariate analysis prompted us to create a score with the two selected risk factors. To evaluate the clinical significance of each risk factor taken separately and of their combination, we investigated the rate of resistance in patients according to the number of risk factors (figure 2). The rates

TABLE 4 Clinical findings at the time of diagnosis of pneumonia in the learning and validation cohorts according to the presence of bacteria resistant to limited-spectrum antimicrobial therapy ${ }^{\#}$

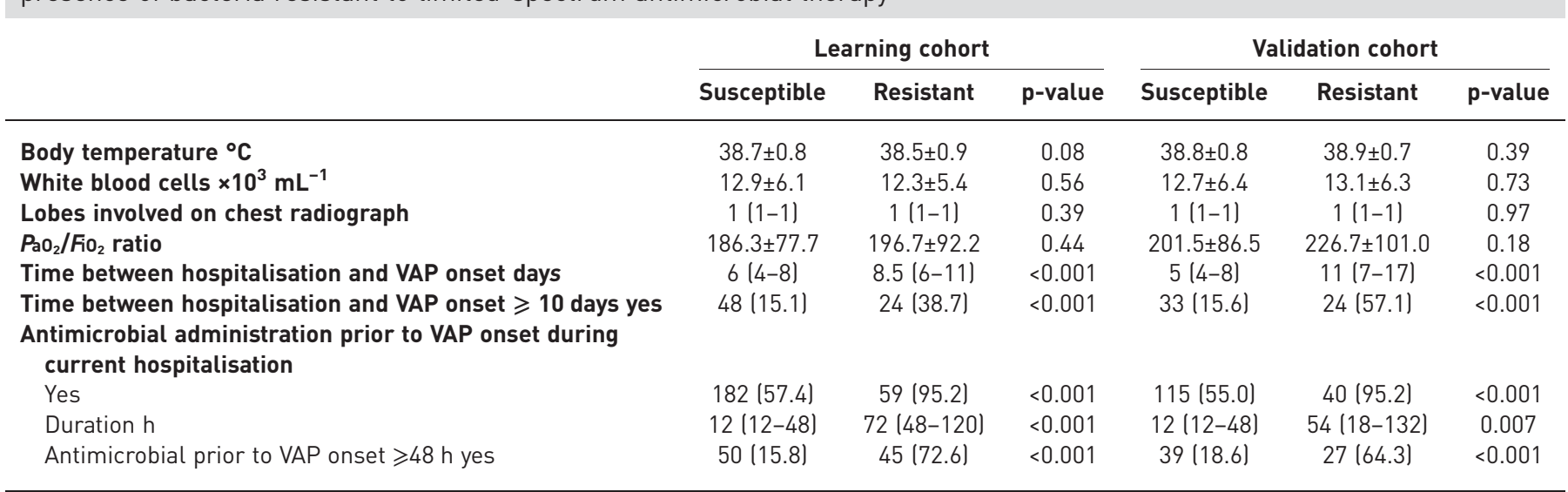

Data are presented as mean $\pm \mathrm{SD}$, median (interquartile range) or $\mathrm{n}(\%)$, unless otherwise stated. $\mathrm{PaO}_{2}$ : partial oxygen tension; FiO 2 : inspiratory oxygen fraction; VAP: ventilator-associated pneumonia. " : ampicillin/sulbactam, ceftriaxone or levofloxacin. 

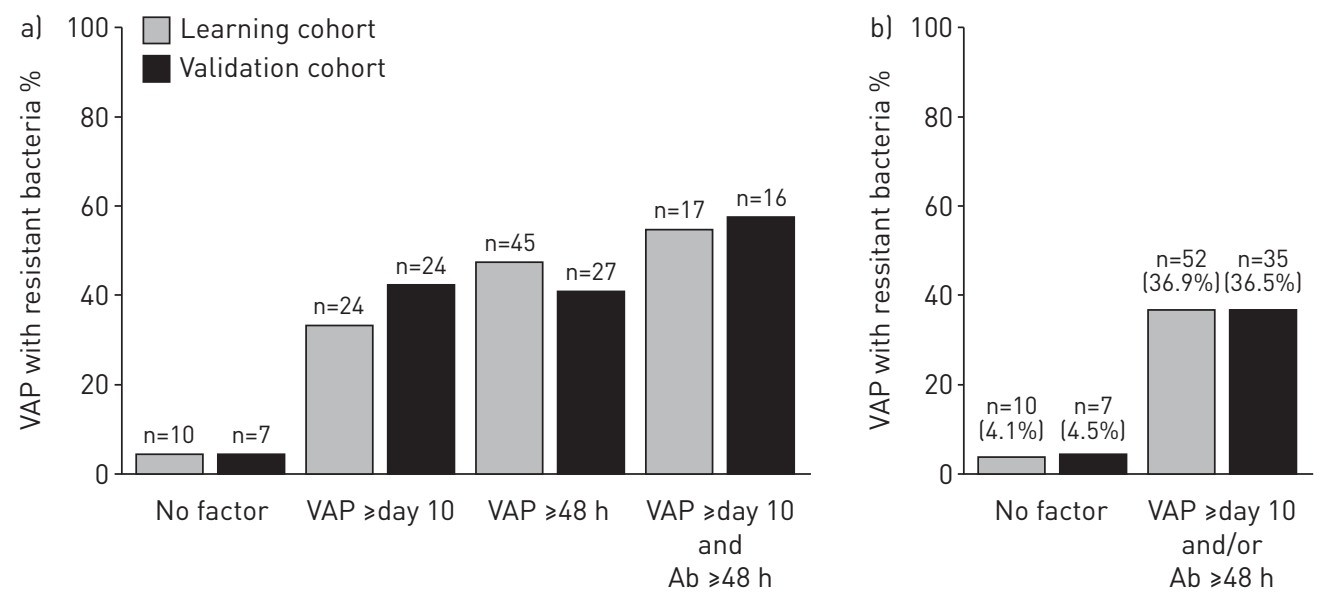

FIGURE 2 Characteristics of the simplified score in the three independent cohorts. Percentage of bacteria resistant to limited-spectrum antimicrobial therapy in the learning and validation cohorts according to a) the number of risk factors and b) the simplified score. VAP: ventilator-associated pneumonia; Ab: prior antibiotherapy administration.

of bacteria resistant to limited-spectrum antimicrobial therapy was $4.5 \%$ in patients with no risk factor and was significantly higher in patients with one or two risk factors (33-47.4\%). The simplified score was constructed as follows: patients with no factor were considered at low risk of resistant bacteria and patients with one and/or two factors were considered at high risk. The characteristics of the simplified score are provided in the table 5. The ability of the score to predict meticillin-resistant Staphylococcus aureus (MRSA) was lower than for prediction of resistant Gram-negative bacteria (online supplementary table S3). When compared with the ATS/IDSA criteria, the net reclassification improvement with the simplified score was $39.1 \%$ in the learning cohort and $35.7 \%$ in the validation cohort. Among the 252 patients of the validation cohort, 185 (73.4\%) were well classified (true positive or true negative) with the simplified score versus $82(32.5 \%)$ with the ATS/IDSA criteria $(\mathrm{p}<0.001)$. Among the 210 patients free of resistant bacteria, $60(28.6 \%)$ would have received unnecessary broad-spectrum antimicrobial therapy with the simplified

TABLE 5 Clinical performance of the simplified score in predicting bacteria resistant to limited-spectrum antimicrobial therapy in the learning and the validation cohorts

ATS/IDSA criteria ${ }^{\#}$

Simplified score ? $^{\text {sin }}$

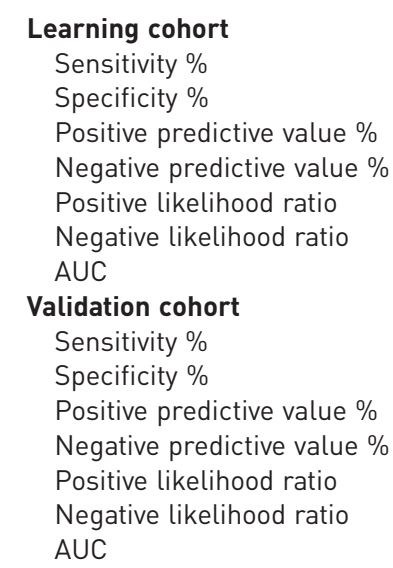

$\begin{array}{cc}100 & 84 \\ 18 & 74 \\ 19 & 38 \\ 100 & 96 \\ 1.2 & 3.2 \\ 0.0 & 0.2 \\ 0.735 & 0.822\end{array}$

ATS/IDSA: American Thoracic Society/Infectious Diseases Society of America; AUC: area under the receiver operating characteristic curve. ${ }^{\#}$ : in the case of late-onset pneumonia ( $\geqslant$ day 5 ), previous antimicrobial therapy in the last 90 days or healthcare-associated pneumonia (hospitalisation for $\geqslant 2$ days in the preceding 90 days, home wound care or residence in an extended care facility, home infusion therapy including antibiotics, or chronic dialysis within 30 days); " : in the case of late-onset pneumonia ( $\geqslant$ day 10 ) or of previous antimicrobial administration $\geqslant 48 \mathrm{~h}$. 
score (false positive, wrongfully classified as resistant) as compared with 170 (81.0\%) with the ATS/IDSA criteria $(\mathrm{p}<0.001)$.

\section{Extrapolation to burn patients}

Finally, to examine if the simplified score could be extrapolated to other ICU populations or should be used only in brain-injured patients, we assessed its predictive values in severe burn patients. Since burn patients were from the same geographic area and from the same hospitals, we considered that any potential differences could not be explained by local variation in ecology [19], but rather by the acute condition on admission (burn versus brain injury). The demographic characteristics and pathogens involved in the cohort of severe burn patients are provided in online supplementary tables S4 and S5. Of 221 patients presenting with VAP, $55(24.9 \%)$ had bacteria resistant to limited-spectrum antimicrobial therapy as compared with $16.7 \%$ in the validation cohort of brain-injured patients $(p=0.03)$. The AUC of the simplified score was 0.671 (95\% CI 0.596-0.751) in the burn patients versus 0.805 (95\% CI $0.732-0.877$ ) in the brain-injured patients of the validation cohort (online supplementary figure S2). The sensitivity and the specificity values of the simplified score were low in burn patients (69\% and 60\%, respectively).

\section{Discussion}

In this study we developed and externally validated a clinical score for the prediction of resistance to limited-spectrum antimicrobial therapy in brain-injured patients presenting with VAP. We show that resistant bacteria should be considered when at least one of the two following factors are present: prior antimicrobial administration during current hospitalisation $\geqslant 48 \mathrm{~h}$ and/or VAP occurrence $\geqslant 10$ days after ICU admission. Brain-injured patients with none of these criteria have a low probability of VAP caused by resistant bacteria. Importantly, this score seems to be specific to brain-injured patients, since it cannot be extrapolated to burn patients.

A restrictive approach to broad-spectrum empiric antimicrobial therapy for VAP could theoretically be advocated in circumstances with a low rate of resistance because the rate of patients wrongfully considered with susceptible bacteria (false negative) will be low. Hence, the intermediate rate of resistance in the participating hospitals (incidence per 1000 patient days: 0.2-0.6 for MRSA and 0.2-0.4 for extended-spectrum $\beta$-lactamase [20]) probably contributed to the high negative predictive value of the score (96\%). However, low rate rates of resistance have already been reported in trauma patients independently from the rate of resistance in the community [9]. Accordingly, the rate of resistance was lower in brain-injured patients than in burn patients in the current study. Taken together, these results argue that the rates of resistance of pathogens involved in VAP are lower in brain-injured patients than in other ICU populations and this result is independent of the rate of resistance in the community.

According to previous publications, the rate of bacterial resistance to antimicrobial therapy ranged from $10 \%$ to $50 \%$ ( $20 \%$ on average) $[10,21,22]$, which is consistent with our findings. This large range of resistance rates may be explained by the variations in local ecology [19] and by several definitions of resistance. Previous predictive scores have been developed considering the natural susceptibility of species to antibiotics, but not the acquired nonnatural resistance (e.g. extended-spectrum $\beta$-lactamase) despite the fact that an increasing frequency is observed in the community [23]. The current definition, based on the measured susceptibility to commonly used antibiotics, could be more useful in clinical practice.

As previously described [24], prior antimicrobial administration and the duration of ICU hospitalisation before VAP were associated with resistance, but we significantly altered the respective thresholds described in the current guidelines [5]. Our results can significantly alter clinical practice, notably in surgical ICUs. Indeed, short-course antimicrobial treatment that efficiently prevents surgical infection or VAP [25, 26] can thus be considered at low risk when using the score described here. The 48-h cut-off, which is in line with the demonstration that a 3-day regimen of antimicrobial agents increases the rate of resistance [3], prompted clinicians to limit prophylaxis to a maximal duration of $48 \mathrm{~h}$.

Contrary to previous publications and recommendations [5, 17], we found no association between resistance and significant healthcare (such as previous hospitalisation) in the last 3 months. In a recent large study in Spain, the empirical antimicrobial therapy recommended for community-acquired pneumonia (limited-spectrum) was accurate to treat severe healthcare-associated pneumonia, defined as developing in patients with one of these two risk factors [22]. Moreover, healthcare-associated pneumonia was not associated with resistance of Pseudomonas aeruginosa in ICU patients with VAP [27]. These results suggest that healthcare-associated risk factors should be better defined before any definitive conclusion can be drawn [28]. Notably, colonisation with antimicrobial-resistant bacteria seems to rapidly decrease after hospital discharge, reaching $<50 \%$ after 2 months [29]. Thus, the delay to consider a preceding hospitalisation ( 90 days) as a risk factor of bacterial resistance could possibly be reduced in brain-injured patients. 
The use of the simplified score to guide the initial probabilistic antibiotic therapy could increase the number of patients that may receive limited-spectrum pre-emptive antimicrobial therapy. Only $4 \%$ of patients were mistakenly considered at low risk (false negative, risk of treatment failure) with the simplified score, while the rate of patients receiving unnecessary broad-spectrum antimicrobial therapy (false positive) decreased from $81 \%$ with the ATS/IDSA criteria down to $28.6 \%$ with the simplified score. This decreased rate of unnecessary broad-spectrum antimicrobial therapy could bring some favourable effects: 1) for the community, because it could theoretically decrease the selection of resistant bacteria in the ICU [30], and 2) for each patient, because the use of unnecessary broad-spectrum antimicrobial therapy has been associated with infection relapse in populations with a high rate of meticillin-susceptible S. aureus [31].

The accuracy of the simplified score was poor in burn patients, in which the rate of resistance was higher than in brain-injured patients (24.9\% versus $16.7 \%$, respectively). As the burn and the brain-injured patients were recruited in the same geographical area, the reported differences should not be explained by variations in the local ecology. Demographic data and medical history of brain-injured and burn cohorts were similar (tables 1 and 2, and online supplementary table S3), suggesting that the observed differences in the rate of resistance depend on the condition on admission. Accordingly, the diagnosis on admission has been shown to impact the epidemiology of VAP in a mixed ICU [8]. We thus propose to consider the initial condition (brain injury versus other conditions) when prescribing empirical antimicrobial therapy for VAP. The simplified score can be deemed adequate for brain-injured patients, but the ATS/IDSA criteria should be used in other conditions.

The study has some limitations. First, we considered only VAP with significant bacterial growth. The simplified score needs to be validated in VAP with low bacterial burden. Second, the characteristics of the score were better for the prediction of resistant Gram-negative bacteria than for MRSA. The rate of MRSA was low in our populations and the predictive values would increase with the prevalence of MRSA in the population. Third, the definition of limited-spectrum antimicrobial therapy can impact the prognostic value of the score. Limited-spectrum antimicrobial therapy was defined as the use of ampicillin/sulbactam, ceftriaxone or levofloxacin because penicillins are the most frequently used antibiotics for treating VAP in Europe [32], and fluoroquinolones are commonly considered as an alternative to penicillin in case of allergy and are recommended by the ATS/IDSA [5]. Ertapenem was not considered here because of its broad spectrum of activity on Enterobacteriaceae and the theoretical risk of resistance associated with the use of carbapenem. Fourth, one could say that $26.6 \%$ of the patients are wrongfully classified, mainly false-positive diagnoses that could lead to unnecessary broad-spectrum antimicrobial therapy. Since the empirical therapy usually lasts $<3$ days, a strategy of de-escalation could limit the risk of spreading resistance in the community [33]. Fifth, this study was performed in five ICUs from three French hospital and the generalisability of its results to other geographic areas, notably those with high rates of resistance in the community, need to be investigated. Finally, this score needs to be validated in a prospective randomised clinical trial. However, two of the participating ICUs have previously reported an increased success rate after implementation of such a score in clinical practice [11].

To conclude, we developed and validated an easy-to-use score predicting bacterial resistance to limited-spectrum antimicrobial therapy in brain-injured patients with VAP. The risk of failure when using limited-spectrum empirical antibiotic treatment is low if VAP onset before day 10 of hospitalisation and if prior antimicrobial administration lasts $<48 \mathrm{~h}$. As compared with the ATS/IDSA criteria, the simplified score could significantly decrease the use of unnecessary broad-spectrum antimicrobial therapy in this specific population and thus limit the spread of antimicrobial resistance. The development of personalised antimicrobial therapy based on the initial condition, i.e. the use of different pre-emptive antimicrobial therapies in patients with different diagnosis on admission even if they are exposed to the same bacterial ecology, appears mandatory.

\section{Acknowledgement}

We thank Dr Hamish McWilliam (Melbourne University, Australia) for his technical support.

\section{References}

1 Vincent JL, Rello J, Marshall J, et al. International study of the prevalence and outcomes of infection in intensive care units. JAMA 2009; 302: 2323-2329.

2 Iregui M, Ward S, Sherman G, et al. Clinical importance of delays in the initiation of appropriate antibiotic treatment for ventilator-associated pneumonia. Chest 2002; 122: 262-268.

3 Bouza E, Granda MJP, Hortal J, et al. Pre-emptive broad-spectrum treatment for ventilator-associated pneumonia in high-risk patients. Intensive Care Med 2013; 39: 1547-1555.

4 Fauci AS, Marston LD. The perpetual challenge of antimicrobial resistance. JAMA 2014; 311: 1853-1854.

5 American Thoracic Society, Infectious Diseases Society of America. Guidelines for the management of adults with hospital-acquired, ventilator-associated, and healthcare-associated pneumonia. Am J Respir Crit Care Med 2005; 171: 388-416.

6 Braykov NP, Morgan DJ, Schweizer ML, et al. Assessment of empirical antibiotic therapy optimisation in six hospitals: an observational cohort study. Lancet Infect Dis 2014; 14: 1220-1227. 

multidrug-resistant pneumonia in intensive care: an observational, multicentre cohort study. Lancet Infect Dis 2011; 11: 181-189.

8 Agbaht K, Lisboa T, Pobo A, et al. Management of ventilator-associated pneumonia in a multidisciplinary intensive care unit: does trauma make a difference? Intensive Care Med 2007; 33: 1387-1395.

9 Kashuk JL, Moore EE, Price CS, et al. Patterns of early and late ventilator-associated pneumonia due to methicillin-resistant Staphylococcus aureus in a trauma population. J Trauma 2010; 69: 519-522.

10 Seguin P, Laviolle B, Dahyot-Fizelier C, et al. Effect of oropharyngeal povidone-iodine preventive oral care on ventilator-associated pneumonia in severely brain-injured or cerebral hemorrhage patients: a multicenter, randomized controlled trial. Crit Care Med 2014; 42: 1-8.

11 Roquilly A, Cinotti R, Jaber S, et al. Implementation of an evidence-based extubation readiness bundle in 499 brain-injured patients. a before-after evaluation of a quality improvement project. Am J Respir Crit Care Med 2013; 188: 958-966.

12 Asehnoune K, Seguin P, Allary J, et al. Hydrocortisone and fludrocortisone for prevention of hospital-acquired pneumonia in patients with severe traumatic brain injury (Corti-TC): a double-blind, multicentre phase 3, randomised placebo-controlled trial. Lancet Respir Med 2014; 2: 706-716.

13 Elm von E, Altman DG, Egger M, et al. The Strengthening the Reporting of Observational Studies in Epidemiology (STROBE) statement: guidelines for reporting observational studies. PLoS Med 2007; 4: e296.

14 Mosier MJ, Pham TN. American Burn Association Practice guidelines for prevention, diagnosis, and treatment of ventilator-associated pneumonia (VAP) in burn patients. J Burn Care Res 2009; 30: 910-928.

15 Magill SS, Klompas M, Balk R, et al. Developing a new, national approach to surveillance for ventilator-associated events: executive summary. Chest 2013; 144: 1448-1452.

16 Lambert M-L, Suetens C, Savey A, et al. Clinical outcomes of health-care-associated infections and antimicrobial resistance in patients admitted to European intensive-care units: a cohort study. Lancet Infect Dis 2011; 11: 30-38.

17 Kollef MH, Shorr A, Tabak YP, et al. Epidemiology and outcomes of health-care-associated pneumonia: results from a large US database of culture-positive pneumonia. Chest 2005; 128: 3854-3862.

18 Hippisley-Cox J, Coupland C. Predicting risk of upper gastrointestinal bleed and intracranial bleed with anticoagulants: cohort study to derive and validate the QBleed scores. BMJ 2014; 349: g4606.

19 Rello J, Sa-Borges M, Correa H, et al. Variations in etiology of ventilator-associated pneumonia across four treatment sites: implications for antimicrobial prescribing practices. Am J Respir Crit Care Med 1999; 160: 608-613.

20 Carbonne A, Arnaud I, Maugat S, et al. National multidrug-resistant bacteria (MDRB) surveillance in France through the RAISIN network: a 9 year experience. I Antimicrob Chemother 2013; 68: 954-959.

21 Martin-Loeches I, Deja M, Koulenti D, et al. Potentially resistant microorganisms in intubated patients with hospital-acquired pneumonia: the interaction of ecology, shock and risk factors. Intensive Care Med 2013; 39: $672-681$.

22 Vallés J, Martin-Loeches I, Torres A, et al. Epidemiology, antibiotic therapy and clinical outcomes of healthcare-associated pneumonia in critically ill patients: a Spanish cohort study. Intensive Care Med 2014; 40: $572-581$.

23 Doi Y, Park YS, Rivera JI, et al. Community-associated extended-spectrum $\beta$-lactamase-producing Escherichia coli infection in the United States. Clin Infect Dis 2013; 56: 641-648.

24 Trouillet JL, Chastre J, Vuagnat A, et al. Ventilator-associated pneumonia caused by potentially drug-resistant bacteria. Am J Respir Crit Care Med 1998; 157: 531-539.

25 Liberati A, D'Amico R, Pifferi S, et al. Antibiotic prophylaxis to reduce respiratory tract infections and mortality in adults receiving intensive care. Cochrane Database Syst Rev 2009; 4: CD000022.

26 Roquilly A, Marret E, Abraham E, et al. Pneumonia prevention to decrease mortality in intensive care unit: a systematic review and meta-analysis. Clin Infect Dis 2015; 60: 64-75.

27 Kollef MH, Chastre J, Fagon J-Y, et al. Global prospective epidemiologic and surveillance study of ventilator-associated pneumonia due to Pseudomonas aeruginosa. Crit Care Med 2014; 42: 2178-2187.

28 Ewig S, Welte T, Chastre J, et al. Rethinking the concepts of community-acquired and health-care-associated pneumonia. Lancet Infect Dis 2010; 10: 279-287.

29 Haverkate MR, Derde LPG, Brun-Buisson C, et al. Duration of colonization with antimicrobial-resistant bacteria after ICU discharge. Intensive Care Med 2014; 40: 564-571.

30 Salgado CD, O Grady N, Farr BM. Prevention and control of antimicrobial-resistant infections in intensive care patients. Crit Care Med 2005; 33: 2373-2382.

31 Chang F-Y, Peacock JE Jr, Musher DM, et al. Staphylococcus aureus bacteremia. Medicine 2003; 82: 333-339.

32 Rello J, Lipman J. Antibiotic prescription for respiratory tract infections in ventilated patients: where are we heading? Intensive Care Med 2013; 39: 1644-1646.

33 Rello J, Chastre J, Cornaglia G, et al. A European care bundle for management of ventilator-associated pneumonia. J Crit Care 2011; 26: 3-10. 